Euskal ikerketen aldizkaria | Revue d'études basques |

Revista de estudios vascos | Basque studies review

$1 \mid 1996$

Numéro I

\title{
Etxahun et la langue française
}

Jean Haritschelhar

\section{OpenEdition \\ Journals}

Édition électronique

URL : http://journals.openedition.org/lapurdum/1876

DOI : 10.4000/lapurdum.1876

ISSN : 1965-0655

Éditeur

IKER

Édition imprimée

Date de publication : 1 octobre 1996

Pagination : 121-139

ISBN : 2-84127-106-4

ISSN : $1273-3830$

\section{Référence électronique}

Jean Haritschelhar, "Etxahun et la langue française », Lapurdum [En ligne], 1 | 1996, mis en ligne le 01 septembre 2010, consulté le 01 février 2020. URL : http://journals.openedition.org/lapurdum/1876 ; DOI : 10.4000/lapurdum.1876 
Jean HARITSCHELHAR

\section{ETXAHUN ET LA LANGUE FRANÇAISE}

Alors que la majorité des paysans basques était unilingue basque au $\mathrm{XIX}^{\circ}$ siècle, bien peu d'entre eux allant à l'école, leurs parents estimant qu'ils pouvaient rendre service à la ferme, il semblerait que le poète Etxahun de Barcus ait fréquenté l'école dans son enfance. Il y fait allusion dans une strophe de la "Chanson de sa vie".

Çortci guerren ourthia nian escolaco

nic hanere çorthia, etchen beçalaco ${ }^{(1)}$

Il dut y apprendre à lire, à écrire et à compter et certainement un minimum de français qui lui permettrait plus tard de s'entretenir avec des personnes non bascophones qu'il aurait l'occasion de fréquenter juges, avocats, procureurs du roi, gens de l'administration pénitentiaire, etc. - au cours de nombreux procès, un en correctionnelle et trois en assises et, bien entendu, dans d'autres occasions.

\section{Le français oral}

Les procès d'assises nous apportent la preuve de sa connaissance du français oral, même si les propos qu'il tient sont vraisemblablement pensés en basque et énoncés en français. Lors du procès devant la Cour d'assises de Pau, en août 1828, le journaliste du Mémorial béarnais fait remarquer que plusieurs des témoins ne parlent pas la langue française et que le sieur Pierre Iritcity, déjà nommé pour l'interrogatoire d'Etxahun, fait encore office d'interprète pour le procès.

Ce procès est capital pour Etxahun car, accusé de tentative d'assassinat commis avec préméditation et de guet-apens, le poète risque la peine de mort. C'est pourquoi il se défend lui-même comme un beau diable, se passant de l'interprète et s'exprimant en français. Le journaliste du Mémorial Béarnais en est témoin : "Après que les diverses formalités prescrites par la loi ont été remplies, il (Etxahun) se lève et répond avec précision à toutes les questions qui lui sont adressées. Peu familiarisé avec la langue française, il emprunte des circonlocutions et des images hardies à l'idiome basque et son langage gagne à ce mélange je-ne-sais-quoi d'original et d'énergique." (2)

Quelque dix-sept années plus tard, en 1845, on retrouve Etxahun devant les Assises des Basses-Pyrénées où il est condamné à trois ans de prison pour faux en écriture publique, jugement cassé par la Cour 
de cassation qui le renvoie devant la Cour d'assises des Landes devant laquelle il comparaît en novembre 1845. Le journaliste du Journal des Landes nous apporte son témoignage : "Pierre Topet ne veut pas être condamné sans être entendu. Sa langue est pourvue au moins d'autant d'activité que ses jambes... Il prend la parole et raconte son histoire ou plutôt son roman avec un accent impossible à décrire et qui donne à son récit quelque chose de pittoresque et de parfaitement amusant. "J'ai eu des malheurs domestiques s'écrie Topet avec une excessive volubilité, ma femme avait cinq ou six amants, je résolus de me retirer dans un ermite (sic)."(3)

La connaissance du français oral est ainsi attestée chez Etxahun qui a appris à lire à l'école, y compris le français. Nous en avons la preuve quand, en 1850 , alors que le poète a 64 ans, il se fait attaquer une nuit dans son logement de Garindein, attaque au cours de laquelle il perd un œil et on le cambriole. Il déclare, au cours de sa déposition, qu'on lui a dérobé un certain nombre d'objets parmi lesquels se trouvent "les cinq codes en un volume, une grammaire latine, deux sacs en toile avec un grand étui en fer blanc dans lequel étaient plusieurs contrats..." (4)

On ne s'étonnera pas de voir Etxahun posséder les "cinq codes en un volume", sachant combien, pendant toute sa vie, il s'est dédié à la chicane, mais on ne peut que s'interroger sur l'existence d'une grammaire latine qui marque, de la part d'un paysan basque, un désir de culture envers ce qui était considéré comme la langue noble par excellence.

\section{Le français écrit}

Grâce à $\mathrm{M}^{\mathrm{me}}$ Péria qui en a fait don au Musée basque, nous possédons des manuscrits d'Etxahun qui prouvent qu'il savait écrire, non seulement en basque (divers poèmes) mais aussi en français. J'ai, en effet, publié plusieurs documents dans mes thèses de doctorat eslettres:

1) La lettre adressée par Etxahun au procureur du Roy, le 1 août 1832, dans laquelle il demande que sa femme soit enfermée dans une maison de force. Elle est ainsi libellée, alors que je l'avais transcrite avec quelques infimes erreurs dans ma thèse ${ }^{(5)}$ :

"Monsieur, Monsieur le procureur du Roy,

Vote ecxelence,

Moy pierre topet dit Etchahon cultivatur à Barcux je viens a vous plaindre de ma situation fatale, quant a vous je ne saurais mais quant a vos adcedens on ne ignoroit pas j'été frapé du plusieurs malheur de part d'une femme infidèle $1^{\circ}$ - d'un long empisonement $2^{\circ}$ - d'un fruit de sa infidélité qu'elle avoit conçue pandant mon abssence et $3^{\circ}$ - de la distruction de mes Biens qu'elle m'avoient distruit pandant mon absance, soit mais bétails qu'ils valoit du Moins $2000 \mathrm{f}$ soit mes linges qu'il valoit du moins $1200 \mathrm{f}$ tous les arbres 
meme mes habillements. Les Bons planches Bons soulives des soules ou de fenils, a la fin tout ce qu'elle pourra attraper sur le moment aussi comme je vins a racheter ma fonds d'etchahon vandue fausement a cause de ses scandales qu'ils m'ont joui un long temps sans aucun droit par concience dont la Justice ma rendu en propriété qu'il ma couté du moins $3000 \mathrm{f}$ d'entrer en possession, elle voyant les récoltes m'appartienoie elle me commence de prodiguer mon Bien en vendant pandant mon abssence tout ce que je puis recuillir des récoltes, dont je voudrois vous suplier pour la faire corriger vuilliez avoir la Bonté de la faire rentrer a une maison de force pour un certain temps, comme il ma impossible d'entretenir mes enfens et mes obligations en sa compagnie et et elle ma rendu trop pauvre pour luy entretenir un penssion dont je voudrois vous supplier s'il y a Lieu de La faire arreter si tot possible

A S. Palais, le $1^{\circ}$ oust 1832 avec Le plus profond respect par votre estimable perssone

votre servitur $\mathrm{Mr}$ le procurur du Roy

topet dit etchahon"

2) Un prologue en français au texte d'une chanson basque en 49 couplets adressée au Procureur du Roy près le tribunal correctionnel de Saint-Palais, M. Clérisse, document que l'on peut dater de $1834^{(0)}$ :

"Monsieur Le procureur du roy il y a queque temps que vous m'avois un honneur singulier en me faisant coumparetre a devant un parisien n'étant capable de vous satisfer, alors je vous avois promis de vous donner L'histoire de ma vie par chacons Basques avec La traduction francoise dont je vous tardé de tenir ma promesse jusque Le momant, connessant indigne de coumparaitre devant vos yeux, mais aujourdhy mon incapacité au coté ma necessité me force de jetter a vos pieds en vous demandant une grace en Le dernier versset de mes tristes chanchons."

3) Une sorte d'épilogue en français à la même "Chanson de sa vie", essentiellement explicatif sur la chronologie exprimée : ${ }^{(7)}$

\section{"Monsieur}

Je vous demande excuse le fautes que le hate ma fit faire, quant aux griefs que je les explique pour les sujet de ces coublets je ne rien ajouté plutot diminué et aux années accordées avec les soufences il a put etre quelque changement c'est a dire 2 question ils pourront etre arribés dans un an quoique je les ai mis an par an et je mis le $31^{\mathrm{e}}$ avant le $30^{\circ}$. Si vous soucie de les faire copier vous faires observer.

Monsieur Monsieur j'avoé je né rien mis qu'il soit digne de votre attention ni soufert rien moins que je écrit. 
Je vous salue avec le plus profond respec par votre estimabre personne.

Topet dit Etchahon".

4) Une phrase en français précédant le poème à Ernest Legouvé (Musde Legouvé) ${ }^{(8)}$

"Monsieur Monsieur le procurur du roi les voila le copie des chansons que je fites par monsieur Legouvé votre cher ami le 20 octobre 1833."

5) Une phrase à la fin du même poème ${ }^{(9)}$ :

"Monsieur Monsieur le procurur du roy je vous le lesser aux mains tierces pour vous faire confus de mon ignorence a presence de votre estimable personne.

Le 29 octobre 1833

votre incapable serviteur

topet dit echahon."

6) Une phrase au début du poème Musde Clérisse ${ }^{(10)}$ :

"Monsieur Monsieur le Procurur, je vous les ai refet vos chançon perssumant dans lesquelles j'avois mis certin vers trop indecen par votre honorable perssone."

7) Un écrit assez important à la fin du même poème :(11)

"Monsieur monsur la hate ma baucup de faute et rature et je ne pas pis du temps a les refere ; comme vous le scavez j'etois alé le 24 du courant et a falu rester la jusque le 28 et quant aux chançons faites en ma propre je vous les envoyerez si tot possible ayant fait chez moy et qui que ce soit vous demandez de moy vous suffira de me marquer, je serez journelement votre servitur et je ne serez comment vous remercier vous m'avoir fait avoir du sieur Legouvé $20 \mathrm{f}$ votre très humble servitur pour ma vie si je suis capable pour rien.

Je vous salue, topet dit etchahon"

Quand on examine ces textes du point de vue du français, il y a beaucoup à dire sur l'orthographe, mais il serait malséant d'insister quand on songe que la scolarité du poète a dû être courte et certainement fragmentaire comme cela était fréquent jusqu'au début de la seconde guerre mondiale, le travail de la maison primant sur l'école. On trouve quelques oublis de consonnes : "vote" mais plus loin "votre", "queque" mais "quelque" dans un autre texte, "empisonement". 
D'autre part, Etxahun écrit comme il parle, c'est-à-dire qu'il ne sépare pas toujours les mots, en particulier avec les auxiliaires être et avoir: "j'été frapé", "dont la justice ma rendu en propriété qu'il ma couté du moins 3000 f." En fait, Etxahun a certainement copié ses manuscrits avec quelque hâte et il en a conscience puisqu'il s'en excuse auprès du procureur du Roy. Il est vrai qu'il oublie la cédille dans le mot "chancon" (pour chanson) qu'il écrira quelques lignes plus loin "chanchon".

Par contre, il est logique avec lui-même quand il écrit "procurur", plusieurs fois, ainsi que "cultivatur" ou "servitur". En effet, les mots se terminant en français par "eur" se terminent par "ür" en souletin (controleur devient kuntrulür dans Eihartxe eta Mignau) et même s'il écrit "malheur" dans sa lettre du $1^{\text {er }}$ août 1832, il écrit "malurra" dans le dernier vers de la sixième strophe de Mundian malerusik:

Bai etare causatu galtceko malurra.

L'euskara ignorant le genre, il est normal que des erreurs soient commises en masculinisant des noms féminins et vice versa. C'était d'ailleurs un des ressorts comiques destiné à ridiculiser les Basques dans un certain théâtre volontiers critique envers eux. Etxahun ne déroge pas à la règle et l'on cueillera au passage "sa infidélité", "ma fonds d'Etchahon", "un penssion", "le fautes" - encore que l'on peut penser qu'il a voulu écrire "les fautes" en oubliant l'accent - "le hate", etc.

En réalité, on a affaire à une pensée en basque exprimée en français. Quelques exemples le prouvent. Ainsi, quand il écrit "je viens a vous plaindre de ma situation fatale", il pense : "horra nitzaizü", c'està-dire "je viens vers vous pour me plaindre". Quelques lignes plus loin, stigmatisant sa femme, il déclare : "elle me commence a prodiguer" qui correspond dans sa pensée à "hasten zait". Dans le deuxième document, il écrit "ma nécessité me force de jetter a vos pieds" ce qui sous-entend le verbe "behartzen nai" et quand il avoue avoir "tardé de tenir ma promesse jusque le moment" on peut supposer un "orai artio".

Autre exemple significatif : "comme je viens a racheter ma fonds d'Etchahon... qu'ils m'ont joui un long temps", la proposition relative équivaut au basque "lüzaz gozatü deitaiena".

Dans des textes aussi courts, on ne peut qu'être surpris par l'emploi du relatif "dont" qui revient quatre fois. L'emploi est tout à fait normal lorsqu'il écrit au sujet de sa propriété : "je viens a racheter ma fonds d'Etchahon... dont la Justice ma rendu en propriété" puisque "dont" a pour antécédent le mot "fonds".

Dans le deuxième cas, il accumule les griefs contre sa femme et poursuit : "dont je voudrois vous suplier..." En réalité, "dont" est ici mis à la place de "ce dont" ou encore "ce pourquoi".

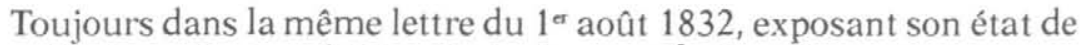
pauvreté et son incapacité à entretenir ses enfants et à verser une pension à sa femme, il conclut : "dont je voudrois vous supplier..." en résumant dans ce "dont" toutes les raisons qui précédent et ici encore "dont" correspond à "c'est pourquoi", "c'est pour cela". 
Plus difficile à expliquer est l'emploi de "dont" dans le quatrième cas : "je vous avois promis de vous donner L'histoire de ma vie par chacons Basques avec La traduction francoise dont je tardé de tenir ma promesse..." Il est évident que dans l'esprit d'Etxahun "dont" fait allusion aux promesses faites de la chanson basque et de sa traduction. Il écrit "dont" au lieu de "et de cela".

Il semblerait qu'au cours de ses lectures de type juridique, certainement, et de ses rapports avec avocats, juges, notaires et huissiers n'oublions pas qu'en 1832, outre quelques comparutions devant le juge de paix, des procès au civil avec les membres de sa famille, il a connu aussi un procès en correctionnelle puis en appel pour l'affaire Goyhenx et le procès en assises de 1828 - Etxahun se soit familiarisé avec une certaine langue où l'emploi du relatif "dont" devait être fréquent, et qu'il a été attiré aussi par l'expression "rien moins que", qu'il emploie à la fin de l'épilogue de la "Chanson de sa vie" où il écrit : "J'avoe je né rien mis qu'il soit digne de votre attention ni soufert rien moins que je écrit", où ce "rien moins que" correspond à "pas moins que".

Dans le droit fil de ce compagnonnage avec la langue juridique, on retiendra la précision de l'emploi de certains termes : si "comparaître" lui est familier avec tous ses démêlés avec la justice, le mot "adcedens" ne peut avoir été recueilli que dans des actes notariés, exploits d'huissiers ou encore jugements au civil.

En fait, avoir avoir cherché vainement dans le Larrousse en dix volumes ou encore le Littré, il a fallu que je me tourne vers le Gaffiot qui, donnant "adsc, voir asc", par suite de la chute normale en latin de la consonne "d", me conduise vers un "ascendens" participe de "ascendo" monter, d'où la valeur de ascendants, c'est-à-dire de prédécesseur dans le texte d'Etxahun.

Le terme "corriger", quand il parle de "faire corriger" sa femme est à prendre dans son sens juridique et il l'a employé en basque dans le poème Mundian malerusik:

Eçagutu nianin cer nian erosi

Nahi ukhen nin bortças corregieraci ${ }^{(12)}$

Corriger, c'est "ramener au bien ce qui est mal, à la règle ce qui s'en écarte, redresser. On corrige les défauts d'une personne et on corrige une personne de ses défauts" (Littré). C'est ce que veut Etxahun et, d'ailleurs, dans le langage juridique, on connaît la maison de correction, "lieu destiné à enfermer par autorité publique les personnes dont la conduite est déréglée et, aujourd'hui, les enfants acquittés pour défaut de discernement" (Littré).

Ce n'est pas une maison de correction qu'Etxahun souhaite pour sa femme mais une "maison de force". Ici encore, admirons la précision du vocabulaire car la maison de force est, selon Littré : " Terme d'ancienne coutume. Maison de force ou maison forcée, prison des femmes de mauvaise vie". Or; pour Etxahun, son épouse est effectivement une femme de mauvaise vie à cause de son inconduite (selon lui, elle avait 
cinq ou six amants) d'où l'emploi du pluriel dans une strophe de Bi berset dolorusik :

Bost urthez presuntegin egon niz inozenki ;
Emaztia xalanteki, harez nintzan jelosi ${ }^{(13)}$

Tels sont les griefs d'Etxahun envers sa femme. Nul ne s'étonnera que le poète connaisse ce terme, essentiellement juridique à l'origine, et qu'il l'emploie à bon escient dans l'épilogue à la "Chanson de sa vie". Un autre terme retient l'attention : c'est l'adjectif "accordées" dans la phrase "et aux années accordées avec les soufences...". Ici, Etxahun veut marquer la correspondance qui existe entre la chronologie et les souffrances qu'il a endurées, ce qui suppose un accord entre le temps et les faits même si, comme il l'avoue lui-même, "il a put être quelque changement..."

Autre formule de type juridique : "Je vous le lesser aux mains tierces" qui explique qu'il ne remet pas le poème Musde Legouvé en main propre à $\mathbf{M}$. Clérisse.

Lorsque Etxahun écrit pour solliciter quelque chose, il sait se faire humble. Le procureur du Roy mérite le titre d'excellence: "Vote ecxelence" et il termine sa lettre du $1^{\text {er }}$ août 1832 , comme la fin de l'épilogue de la "Chanson de sa vie" en 1834, par la même formule : "avec le plus profond respect par votre estimable personne", à quelques nuances orthographiques près, ou encore "honorable personne", formules consacrées ou utilisées couramment à cette époque-là, ce qui prouve qu'Etxahun les avait, soit lues, soit entendues auparavant.

\section{Un document nouveau}

Un document nouveau complète notre documentation sur les rapports entre le poète de Barcus et la langue française. Il s'agit d'un poème en français adressé au préfet des Basses-Pyrénées, de passage à Mauléon, vraisemblablement pour le tirage au sort, le 3 juillet 1840, dans lequel il demande que son fils soit exempté du tirage au sort.

La photocopie de ce document m'a été donnée par M. d'Andurain de Maytie, ambassadeur de France, demeurant à Pau, un des descendants de la famille d'Andurain de Mauléon, qui l'a trouvé dans les archives de la famille. En effet, M. d'Andurain écrit : "Ce document des archives privées d'Andurain a été présenté au Musée basque à l'intention de M. Haritschelhar le 10 juin 1980 par M. d'Andurain de Maytie qui lui adresse ses meilleurs compliments."

Seize années ont passé depuis et ce n'est qu'à l'occasion de cet article que j'exhume ce poème dont je ne pense pas qu'il ajoute grand chose à la renommée d'Etxahun mais qui, cependant, est un nouveau témoignage sur la connaissance du français par le poète. Remarquons que, six ans auparavant, il avait donné la "Chanson de sa vie" en basque (fort heureusement pour nous) et la traduction en français, alors qu'en 1840, c'est directement en français qu'il veut s'adresser à l'autorité et, 
128

Jean Haritschelhar

Als liver be pefet du pau, vici un isnoientqu it na eu que ta Cerveau, rul metréni iogent qui fiait hup vers numeau, par son petit talent

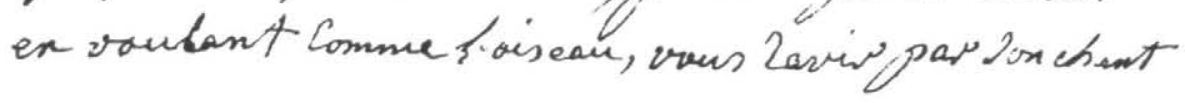

je vous en pelicite, natre prefet raillant de Thonnorabletitre, quivens eminant crest ha Drne corchite qu il saur a mis sigran et ha haule mesite lesotre ilurtre vans

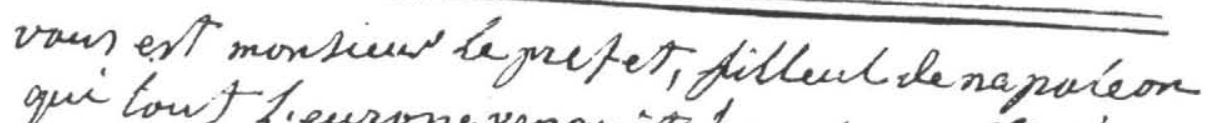
qui tow' lieurope vanquit; homis que tratison vour vous trousce diexpert parratse sunteion ajant terte ce dujett, terpist arec be som

mon prefet huret humain, Dore de but sonnews

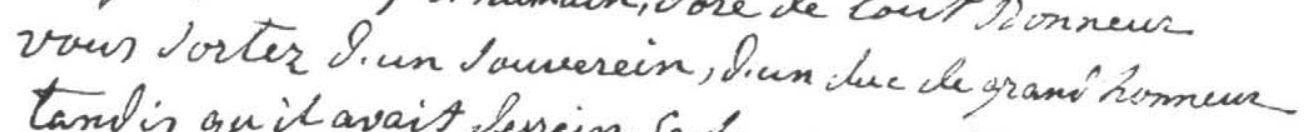
tand is quithavait lessein, Ge-framenp empereus

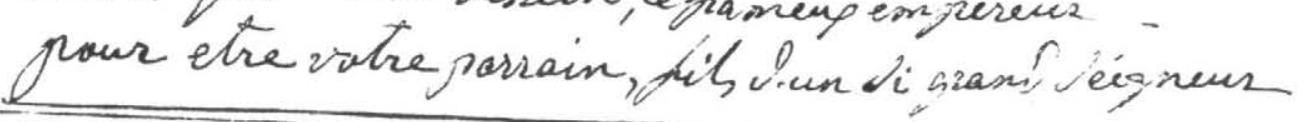
le za rille le Chatel, vous est vesu a pan pour bele qui vous a en, oh quet lare Cackan

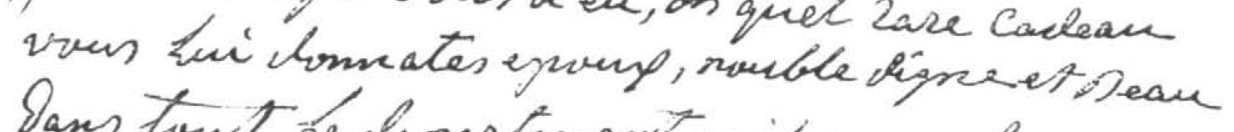
Sans towt ie departemeni, ar il napa vin egear 
Etxahun et la langue française

129

vous aveiz une femme Selon retie veriz

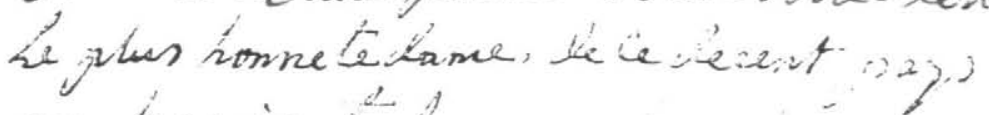

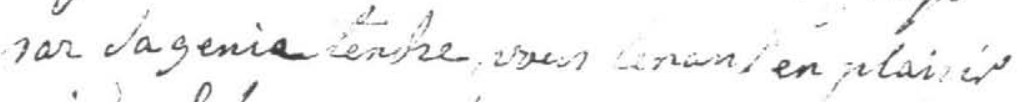
qui end keureup oname t técospen snai

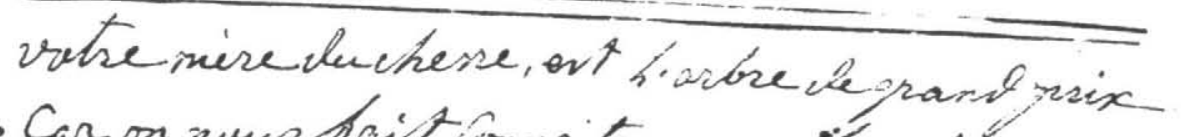

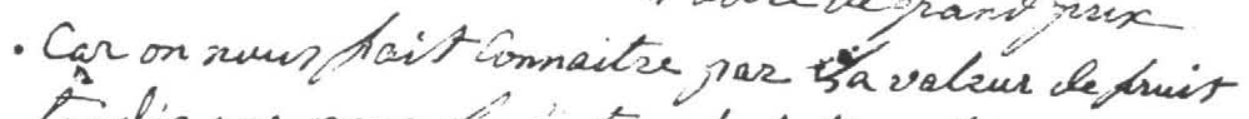

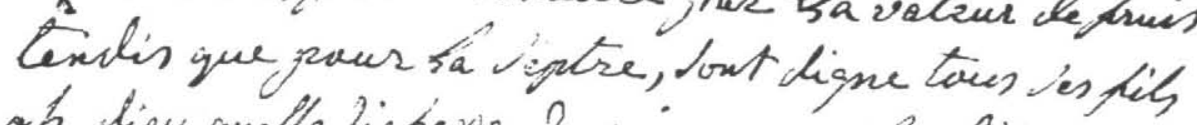

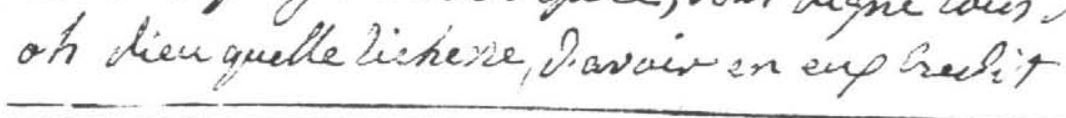

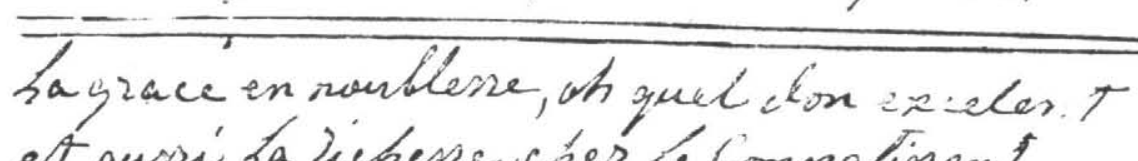
et awiwi ta lichere, cher ke Compaliss an! Ceque sirqueil tes eche, Dans un Caeur inconstant mais avec sous ses practhe, wotre preftes pieinant

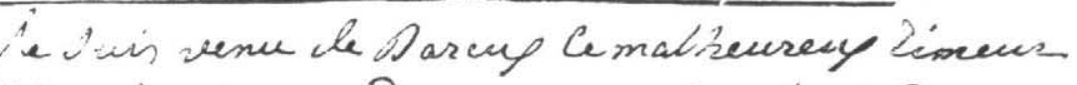

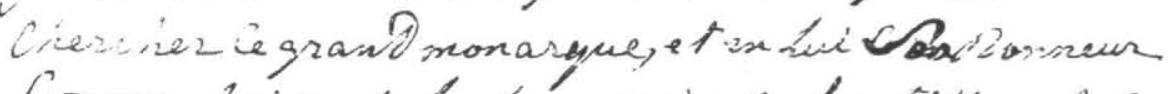

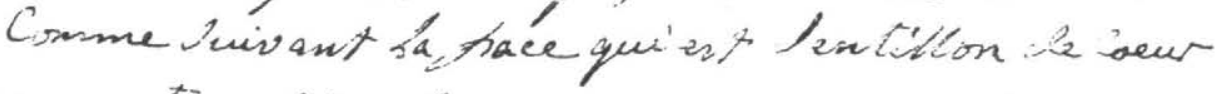
vow des plain begrace, pour usens weinalkeur

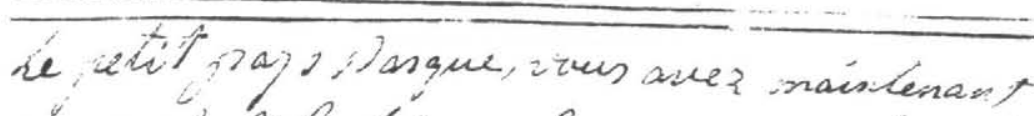

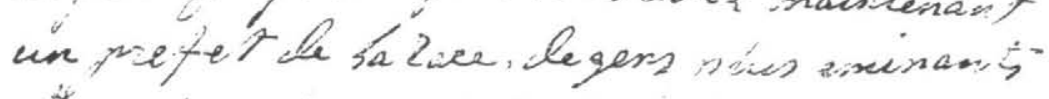

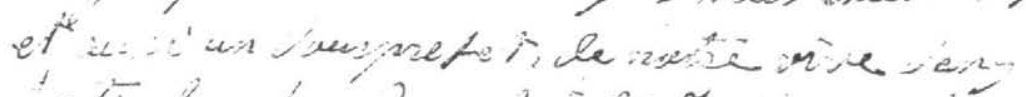

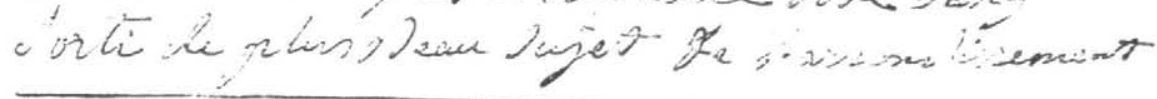


130

Jean Haritschelhar

Hotre Lave 'unnefet, obligent af able

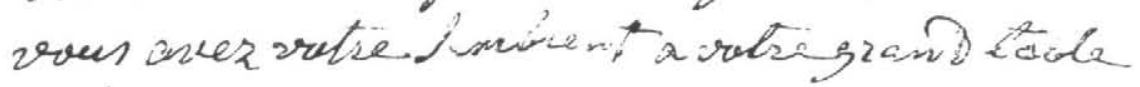

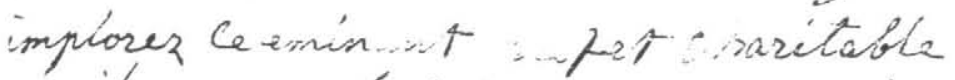

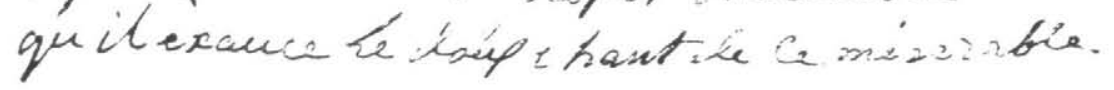

in

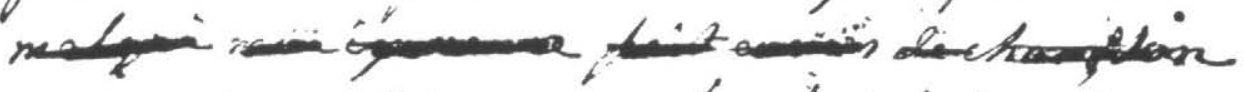

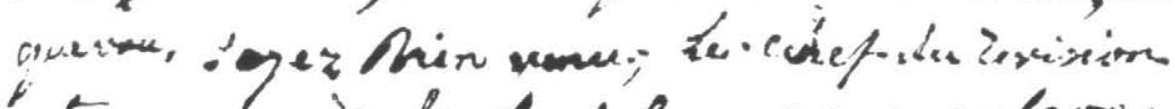

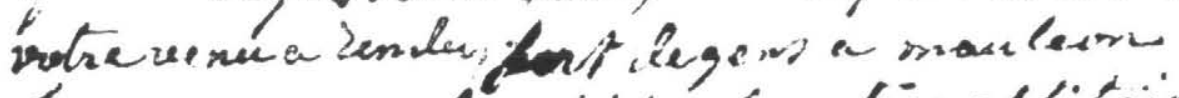

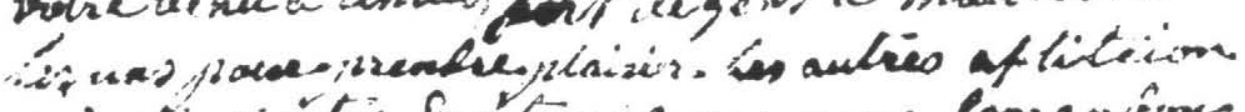

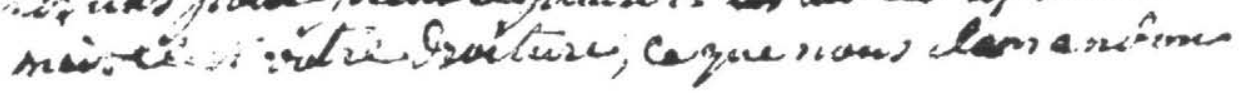
res chan yzix) incapablip mon dyet opulant on ne meritaien gegele reus tro ni argant mais fayais ar tarke, un infirme enfant

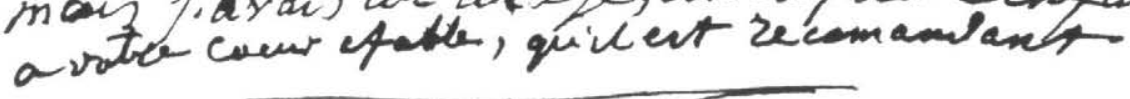

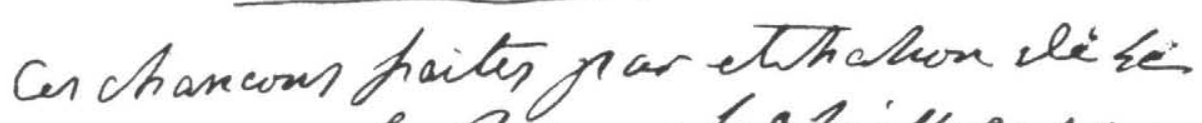
Commune he Dareey Les/nillel eszo 
de plus, en vers. C'est comme au procès d'assises en 1828, il n'a pas besoin d'interprète.

Si ce document se trouve dans les archives d'Andurain, c'est tout simplement parce que, même s'il a été remis au préfet, celui-ci a dû le laisser à M. Clément d'Andurain, sous-préfet de Mauléon. En effet, "par ordonnance du Roi du 19 février 1840, M. d'Andurain (Clément) ancien sous-préfet de Mauléon, est appelé à remplir les mêmes fonctions dans cet arrondissement, en remplacement de M. Etchats, admis à faire valoir ses droits à la retraite. ${ }^{(14) "}$

Examinons la situation d'Etxahun en juillet 1840. Depuis son procès d'assises en 1828, et son pèlerinage à Rome en 1831, il se préoccupe essentiellement de restaurer son patrimoine dispersé pendant sa longue détention. A coups de procès et d'arrangements, il parvient à ses fins, du moins pour une large part. Sa fille aînée, Marie, s'est mariée à Oholegia de Barcus le 7 février 1939, son fils Joseph, destiné à lui succéder à Etxahunia a pris épouse le 19 septembre de la même année. Son fils François, né le 26 avril 1819, a 21 ans en juillet 1840 et c'est certainement lui qui est appelé au tirage au sort et c'est pour lui qu'il sollicite l'exemption. Il faut dire que le poète est certainement au sommet de sa gloire, puisqu'il est appelé à connaître le procureur du roi à SaintPalais, Hippolyte Clérisse, qui lui a fait rencontrer le poète parisien Ernest Legouvé. Il consacre un poème à chacun d'entre eux ${ }^{(15)}$ et la "Chanson de sa vie" au premier. Le 21 juin 1841, il est appelé à Chéraute pour le mariage d'Adélaïde Carricaburu, fille de dame Julie Roger, veuve Carricaburu, native de Barcus et de Jean-Baptiste de Rouilhan domicilié au château de Montaut (Gers) ; il compose un épithalame en l'honneur des jeunes époux et de leurs familles. ${ }^{(16)}$

Le poème est de ceux que l'on peut classer dans les poésies de circonstance et relève du genre laudatif. En effet, Etxahun a une demande précise à faire dans le dernier couplet et il faut qu'il force le compliment afin d'obtenir ce qu'il souhaite. Ce poème est donc à rapprocher des autres du même genre écrits en basque. Dans ma thèse, j'écrivais à leur sujet: "Si l'on compare la poésie laudative à la poésie satirique, on s'aperçoit que le génie inventif du poète est autrement plus habile dans le maniement du fouet de la satire que dans celui de l'encensoir. A la grande diversité des poèmes satiriques s'oppose l'uniformité des poèmes laudatifs. Ils semblent tous faits dans le même moule. Peut-être le genre le veut-il ? Quand on étudie les poèmes d'Etxahun, on découvre rapidement le moule unique dans lequel ils ont été fondus : introduction de compliments, rappel des nobles origines, du lieu de naissance, évocation souvent dithyrambique des nombreuses qualités (une strophe sur la puissance de séduction si l'homme à qui il s'adresse n'est pas marié) conclusion invoquant l'incapacité du poète ou faisant appel à la générosité. Mais si le moule est le même, Etxahun sait tout de même varier ses effets. Nous savons qu'il a suffisamment d'invention pour le faire. Peut-être est-ce dans ce genre qu'il en a le moins. ${ }^{(17) "}$ 
La structure du poème en français relève bien de ce moule unique avec les petites variantes d'usage. La strophe introductive est celle où le poète se présente, humble, ignorant, devant l'autorité préfectorale. Tout concourt à cela : il n'a que des qualités naturelles (que sa cerveau) qui n'ont pu s'épanouir (nul metre ni régent); poète certes qui, s'il est de "petit talent" n'en est pas moins comparé dans le dernier vers, à l'oiseau dont les qualités innées produisent un chant ravissant. Humble sans aucun doute ce poète, mais... ne doutant pas tout à fait de ses qualités.

En général, dans les poèmes laudatifs, l'ignorance et l'incapacité sont exprimées dans la dernière strophe mais pas toujours. Par exemple, dans Musde Legouvé, la première strophe souligne son manque d'esprit par rapport au poète parisien :

Celuco ginco maitia, eguidaçu laguncia nahinuke versetes partu pouetetaco naussia

Eta hari coumparatceco, nic manca spiritia

ainsi que dans Goure Jaun aphescupia, s'inclinant devant l'évêque :

Goure jaun aphescupia, diocesaco princia

çuc guidatcen deicuçuna, erlegione saintia

Indigneric eguindeçut, bi versseten houncia

Nahis hes felicitatu, çuc ukhen verthutia.

La deuxième strophe commence par la phrase : "Je vous en félicite". C'est là une formule consacrée que l'on trouve abondamment dans la poésie laudative d'Etxahun, non seulement dans la citation cidessus mais encore dans Musde Deffis :

Felizitatzen zütüt, Defis jaun jujia (strophe 1)

Hau da berset azkena egiten düdana

Zure felizitatzez, Musde Defis jauna (strophe 10)

dans Üdoien prozesaren khantoria :

Felizitatzen zütüt, Musde Vivie (strophe 5)

Felizitatzen zütüt, Musde Frederik (strophe 8)

dans l'épithalame de Chéraute, Sohütako ezteietan:

Barkoxerik horra niz Sohütara

Ene herritar diñen felizitatzera (strophe 1)

Felizitatzen zütüt kunpaña nublia

Eta zü berheziki Madama Julia (strophe 2)

Felizitatzen zütüt esposa lilia (strophe 5)

dans Musde Chaho :

Barcocheric Bayonara jouitez hountcen dut bi cobla,

Nahiz hayez felicitatu mousde Chaho handen houra

et, enfin, dans Musde Renaud :

Felicitatcen deiçut fama, mousde Renaud (strophe 3) 
Ce qui prouve que le "Je vous en félicite" correspond au "Felizitatzen zütüt" des poésies basques dans le sens de complimenter la personne à qui s'adresse le poète. Dès lors, sont mis en exergue le titre, la conduite, le mérite avec des adjectifs du genre superlatif: "prefet vaillant", "honorable titre", "eminant", "bonne conduite", "si grand", "la haute mérite", "ilustre sang".

Assez souvent, l'ascendance du complimenté est évoquée, parents, lieux de naissance :

- les parents dans Musde Legouvé :

parisen mousde Legouvé obrac agueridirade aita cela mundianceno, liburu famous eguile eta semia verssoulari, uropan pareric gabe (strophe 2)

ou encore dans la version populaire :

Zure aita zen Parisen

Nausi poeta ororen (strophe 4)

dans Musde Clérisse :

Aita duçu hastingaco, juge peira houradaco (strophe 2)

- le lieu de naissance dans Musde Defis :

Defisen sortzepenaz nüzü orai mintzo :

Nik entzünik düdanaz da seme Tarbako (strophe 4)

dans Goure Jaun aphescupia où dans le même vers se trouve évoqué le lieu de naissance et les parents :

Sorthu cinen Rhodasen, seme aitama noublen (strophe 2)

dans Sohütako ezteietan où il est rappelé que le jeune de Rouilhan est né dans la demeure même où vit actuellement la famille dont il épouse la fille

Madamak diozü Durollanti erran :

- "Sorthürik izan zira ni nizan khanberan ;

Nik ere sorthü beitüt zunbait alhaba han,

Hetarik nahi deizüt espusa bat eman"

Pour le préfet, qui s'appelle Napoléon Duchâtel, un illustre parrain est évoqué, en premier l'empereur Napoléon $I^{\text {er }}$, ce qui n'est pas mal venu en cette année du retour des cendres et où la légende touchant l'empereur ne cesse de grandir et de se répandre. Dans le vers :

"qui tout L'europe vanquit, hormis que trahison"

sont étroitement mêlés la gloire de l'empereur et son destin tragique qui reste fixé dans les mémoires ainsi que l'idée qu'un homme aussi invincible ne pouvait être vaincu que par la trahison.

Afin de ne pas déroger à la règle, les parents du préfet font l'objet de deux strophes : le père à la strophe 4

"Vous sortez d'un souverein, d'un duc de grand honneur" 
lui qui est "fils d'un si grand seigneur", la mère à la strophe 7, dame de noble rang puisque duchesse et le poète-paysan n'oubliera pas de mentionner que d'un tel arbre (généalogique) ne peut que naître un fruit de valeur.

En général, Etxahun se renseigne sur le lieu de naissance ou la provenance de la personne qu'il complimente. Nous l'avons vu pour le juge Deffis (Tarbes) comme pour Mgr Lacroix (Rodez). Il est curieux de noter que le poète, à la strophe 5, écrit :

"de la ville de Chatel, vous êtes venu à pau"

car les seuls Chatel existant en France sont de petites communes ou chefs-lieux de canton de Haute-Savoie (la commune de Haute-Savoie n'était d'ailleurs pas française à l'époque), des Vosges ou de Moselle. N'y aurait-il pas eu une confusion dans l'esprit d'Etxahun, le préfet se nommant Napoléon Duchâtel? En effet, de la mêrne manière qu'il écrit : "Monsieur le prefet $d u$ pau", pense-t-il que le préfet est Napoléon $d u$ Chatel?

Dans le schéma laudatif, il convient de mentionner l'épouse du sujet du poème, s'il est marié. Il l'a fait pour le juge Vivié dont il rappelle la fidélité à son épouse dans la strophe 7 de Udoien prozesaren khantoria:

Musde Vivie Jauna balitz andrekari,

Aise egin lirozü hanitxi erri,

Filosomiaz beita ilusigarri

Eta domenanta profetak üdüri ;

Bena eztü nahi

Egin desplazerik

Bere Madamari

Eta ez laidorik zelüko Jaunari

Dans le cas de l'épouse du préfet, elle est "le plus honnete dame de ce décent pays" - toujours du superlatif - et pour Etxahun, dans le mariage, le plaisir de l'âme s'accompagne du plaisir du corps :

"par sa génie tendre vous tenant du plaisir

qui rend heureux son ame et le corps du mari" (strophe 6)

Il est vrai que quand il s'adresse à un célibataire comme le poète Ernest Legouvé, il ne manque pas de faire allusion aux nuits auprès de la future épouse (Musde Legouvé, strophe 9)

jaun gastiac passeiari, çu Legouvé hala ari

Bena halaco andere bat etchen baçunu gaiari

etcinateke tour de françan nahi haboro ebili.

Si nous abordons le thème des qualités - physiques, intellectuelles ou morales - Etxahun devient dithyrambique : le préfet est vaillant, de sang illustre, de bonne conduite (strophe 2), il est doux et humain (strophe 4); en tant qu'époux, il est noble, digne et beau et n'a pas son égal dans tout le département (strophe 5); il possède la grâce en noblesse ainsi que la richesse en sachant être compatissant (strophe 8); 
il est plein de grâce pour les gens de malheur (strophe 9), il est de la race des gens les plus éminents (strophe 10), il est à la fois éminent et charitable (strophe 11) et enfin, un sujet opulent dans la dernière strophe en prenant le terme sujet dans son sens basque de personnage.

Quant à l'épouse, nous l'avons vu, elle est honnête, tendre et sait rendre heureux son mari.

Bien entendu, ces personnages n'ont aucun défaut et le vocabulaire français, celui des adjectifs en particulier, ne diffère pas du vocabulaire basque : "tendrezia", "ümilitatia", "nublezia", "karitatia", "unestatia", telles sont les qualités de Musde Defis; Musde Legouvé est "verssoulari uropan pareric gabe" (pas moins), il est aussi "sutilitatez bethia" et "pariseco sabantena"; les qualités de Musde Clérisse sont "spiritiaren ederra", "umilitatia"; dans Udoien prozesaren khantoria, le juge Vivie est "aberats", "banitate gabe", "afable", la jeune épousée de Sohütako ezteietan est comme son mari "unest eta sajia", et chez ce dernier :

Berthutia denian nubleziareki

Hura düzü ürhia diamantareki

La comparaison est on ne peut plus flatteuse.

L'éloge à Musde Chaho dépasse toute mesure : jeune, savant, vertueux. L'accumulation des qualités se trouve dans la strophe 5 :

Abill, eiger, ezpiritous, dena da ussin handious ;

Bena çu dohain hayegati, etcirade fantesious,

Umil, afable cira eta, behar denian, corajous.

Un éloge du même type est destiné à Musde Renaud, à la strophe 8 :

Aberax, gora, eder, gaste, habil, hardit,

Halacouetan hounest, guti ikhustendit :

Bainan çu mousde Renaud, hala cirelarik

Ezteretçu eguiten, ihouri khorterik,

Arous nahi duçu seguitu Jesus-Christ.

L'évêque de Bayonne, Mgr Lacroix, n'en mérite pas moins. À titre d'exemple, la strophe 5 :

Aphes hounac dirade, avis hounic emaile

Munduco houn plazer eta, ouhoures cassu gabe

Caritatous esti prudent, umil, ounest eta sobre

Mounsignur verthute hoyes çu doraturic cirade.

Comme on le voit, Etxahun a la plume agile quand il s'agit d'encenser et il accumule toutes les qualités sans la moindre vergogne. Si celles-ci sont, à peu de chose près, les mêmes, qu'il s'exprime en basque ou en français, il semble que l'éloge flatteur atteigne son comble dans la pièce en français. Astucieusement d'ailleurs, il associe le sous-préfet d'Andurain à son illustre hôte du jour, d'autant que le sous-préfet est "de notre vive sang, sorti de plus Beau sujet de L'arrondissement". Ce "rare sous-préfet, obligent afable" et à qui on peut s'adresser un jour, n'est pas à négliger: 
Vient enfin la demande ; elle est préparée par la strophe 12 qui fait allusion au tirage au sort, lequel donnera plaisir aux uns, aux autres affliction, selon le poète qui se fera humble à la strophe suivante. Il n'est pas rare qu'à l'ultime couplet, des poèmes laudatifs, Etxahun se reconnaisse incapable comme dans Musde Defis (strophe 10)

Dakidalarik hartaco inkapable nizala

Ene izpiritiaren eskasa dela kausa

ou encore ignorant dans Musde Legouvé (strophe 13)

Mousde Cleriça pharcatu, espaçutut satifatu

pariseco sabantenari aldis erranenderoçu

barcocheco ignorantenac, khantiac deitçola hountu

Ses chansons sont "incapables" aussi devant un "sujet opulant"; il ne demande ni gage, ni or, ni argent, alors qu'un louis serait le bienvenu dans Udoein prozesaren khantoria :

Ni hütsik sakola

Nahigorik lüis bat

Süjet haier beha

qu'il souhaite que ses biens lui soient rendus dans Musde Chaho (strophe 20)

Ene hounen errenderaztia, Bonaparti çutçaz galtheguin,

qu'il sollicite l'aumône de la main de l'évêque dans la strophe 14 de Goure Jaun Aphescupia :

espeitut beste secoursic, gente hounena baici

hartacos cerbait nahi nuke çoure escu saintuti.

Ce qu'il demande au préfet, c'est que son fils présenté comme "un infirme enfant", avec toujours le souci de s'abaisser devant les grands, soit dispensé du service militaire. C'est ainsi qu'il se recommande à un "coeur afable".

Il n'oublie pas que quelques années auparavant, grâce à l'obligeance de mousde maitia, certainement Clément d'Andurain et mousde Etchas, alors sous-préfet ainsi que le président Deffis, il avait pu faire libérer un de ses domestiques qui avait tiré le mauvais numéro au tirage au sort (Chanson de sa vie, strophe 48). Sur sa lancée, à la strophe 49, il sollicitera l'appui du procureur du roi Hyppolyte Clérisse pour faire entrer son fils dans la douane.

Etxahun écrit donc en vers français. Personne ne le savait jusqu'à la publication de ce document. La prosodie française lui est totalement inconnue. Aussi va-t-il composer comme il a l'habitude de le faire en basque. Il utilise la strophe monorime de quatre vers de treize syllabes dans le rythme $7+6$ en marquant la césure par une virgule. S'il n'utilise pas l'alexandrin qui est pourtant le vers majeur de la littérature française, c'est qu'il compose en français comme en basque en utilisant un air connu qui impose le rythme. Il ignore que, en prosodie française, la dernière syllabe est accentuée, dernière lorsque la rime est masculine et avant-der- 
nière lorsque la rime est féminine. La majorité des vers qu'il compose est de rime masculine. Une seule strophe est de rime féminine, mais le $e$ muet compte pour Etxahun car il correspond à la dernière note musicale :

Notre rare sous-préfet, obligent afable

$\begin{array}{lllllllllllll}1 & 2 & 3 & 4 & 5 & 6 & 7 & 1 & 2 & 3 & 4 & 5 & 6\end{array}$

Vous avez votre semblant, a votre grand table $\begin{array}{lllllllllllll}1 & 2 & 3 & 4 & 5 & 6 & 7 & 1 & 2 & 3 & 4 & 5 & 6\end{array}$ implorez ce eminent, prefet charitable $\begin{array}{llllllllllllll}1 & 2 & 3 & 4 & 5 & 6 & 7 & 1 & 2 & 3 & 4 & 5 & 6\end{array}$ qu'il exauce le doux chant de ce misérable
$\begin{array}{lllllllllllll}1 & 2 & 3 & 4 & 5 & 6 & 7 & 1 & 2 & 3 & 4 & 5 & 6\end{array}$

Il n'utilise pas la synérèse car la voyelle finale muette d'un mot compte, même si elle est suivie d'une voyelle :

vous lui donnates époux, nouble digne et beau

ou encore

mais j'avais au tirage un infirme enfant

$1 \quad 2 \quad 3 \quad 4 \quad 567 \begin{array}{lllllll}1 & 2 & 4 & 5 & 4 & 5 & 6\end{array}$

J'ai, dans ma thèse, démontré que le poète de Barcus utilisait l'assonance, à l'instar de ses prédécesseurs. Chez lui compte surtout la dernière voyelle du vers et la consonne qui vient par la suite ne compte pas. Ainsi, $a$ alterne soit avec $a n$, ou $a t$ ou $a k$ ou $a z$ dans la même strophe et il en est de même pour toutes les voyelles. Il utilise aussi la rime portant sur une syllabe entière ou encore une syllabe et demie ${ }^{(18)}$

Il agira de la même manière en français avec des rimes en an,on, eur, $a u, c^{\prime}$ 'est-à-dire des rimes pauvres et il appliquera le principe de la consonne qui ne compte pas après la voyelle finale dans la strophe 6 où désir rime avec pays, plaisir et mari, c'est-à-dire qu'il se contente de l'assonance en $i$. Par contre, la seule strophe à rime féminine est plus riche du point de vue de la rime puisque les quatre vers se terminent en able (afable, table, charitable, misérable).

$\star \star *$

Cette poésie en français n'ajoute rien à la gloire d'Etxahun. Elle n'a d'intérêt que pour mieux saisir sa connaissance de la langue de Molière. J'écrivais dans ma thèse, il y a presque trente ans :

"Les poésies de circonstance ne sont pas un grand fleuron dans l'œuvre d'Etxahun. Le "los" de commande ne l'inspire pas beaucoup. C'est de la poésie que nous avons qualifiée d' "alimentaire" et qu'Etxahun compose comme du travail forcé. Il y est mal à l'aise, beaucoup plus rimailleur que poète". ${ }^{(19)}$

Et ce n'est pas en changeant de langue, en quittant sa langue maternelle qu'il manie avec aisance et en empruntant une langue que je n'hésite pas à qualifier d'étrangère, aussi bien dans son orthographe que 
dans la valeur sémantique des mots qu'il se montrera plus poète que rimailleur. Il ne peut être que rimailleur en français. Cependant, cette chanson est une preuve supplémentaire de la psychologie d'Etxahun. Il est né paysan et il est très attaché à la terre et à l'argent. Sa renommée de poète-improvisateur lui permet d'être en contact avec les "grands" de la petite région. Madré comme il l'est, il se fait alors humble devant ces personnages dont il compte bien tirer profit : ceux du tribunal de SaintPalais, le président Deffis, le procureur du roi Hippolyte Clérisse qui lui présente le poète Ernest Legouvé, le juge Vivié, les avoués Frédéric Darthez ou encore Basterretche, la famille Carricaburu-Roger et les de Rouilhan qui sont célébrés à l'occasion d'un mariage où il est convié, le sous-préfet d'Andurain et le préfet Napoléon Duchâtel et plus tard les hommes politiques de gauche Augustin Chaho et Michel Renaud, enfin l'évêque de Bayonne Mgr Lacroix, à l'occasion d'une confirmation à Barcus. Il n'hésite pas à tresser des lauriers, obtient un louis d'or d'Ernest Legouvé, fait libérer son domestique du service militaire, cherche à faire entrer un fils dans la douane, à faire exempter un autre, quête un louis des chicaniers de Barcus, demande l'aumône à l'évêque de Bayonne. Tel est l'homme, proche de ses intérêts, qui, dans ces caslà, s'avère plus rimailleur que poète.

\author{
Jean HARITSCHELHAR \\ Professeur émérite \\ de l'université Michel de Montaigne - Bordeaux III \\ ERS 142 du CNRS
}




\section{NOTES}

1 - J. Haritschelhar, L'ceuvre poétique de Pierre Topet-Etchahun, Bilbao, 1970, p. 176.

2 - J. Haritschelhar, Le poète souletin Pierre Topet Etchahun (1786-1862), Contribution à l'étude de la poésie populaire basque du XIX* siècle, Amis du Musée basque, Bayonne, 1969, p. 145.

3 - J. Haritschelhar, Le poète souletin Pierre Topet Etchahun (1786-1862), Contribution à l'étude de la poésie populaire basque du XIX'siècle, Amis du Musée basque, Bayonne, 1969, p. 226.

4 - Jean Haritschelhar, Le poète souletin Pierre Topet Etchahun (1786-1862), Contribution à l'étude de la poésie populaire basque du XIX' siècle, Amis du Musée basque, Bayonne, 1969, p. 248.

5 - Jean Haritschelhar, Le poète souletin Pierre Topet Etchahun (1786-1862), Contribution à l'étude de la poésie populaire basque du XIX's siècle, Amis du Musée basque, Bayonne, 1969, (hors texte) et p. 541.

6 - J. Haritschelhar, L'ceuvre poétique de Pierre Topet-Etchahun, Bilbao, 1970, p. 172.

7 - J. Haritschelhar, L'œuvre poétique de Pierre Topet-Etchahun, Bilbao, 1970, p. 188.

8 - J. Haritschelhar, L'œuvre poétique de Pierre Topet-Etchahun, Bilbao, 1970, p. 522.

9 - J. Haritschelhar, L'ceuvre poétique de Pierre Topet-Etchahun, Bilbao, 1970, p. 524.

10 - J. Haritschelhar, L'ceuvre poétique de Pierre Topet-Etchahun, Bilbao, 1970, p. 542.

11 - J. Haritschelhar, L'ceuvre poétique de Pierre Topet-Etchahun, Bilbao, 1970, p. 544.

12 - J. Haritschelhar, L'ceuvre poétique de Pierre Topet-Etchahun, Bilbao, 1970, p. 78.

13 - J. Haritschelhar, L'œuvre poétique de Pierre Topet-Etchahun, Bilbao, 1970, p. 142.

14 - Le Mémorial des Pyrénées, journal de Pau, 10 mars 1840.

15 - J. Haritschelhar, L'ceuvre poétique de Pierre Topet-Etchahun, Bilbao, 1970, p. 522 et p. 542 . p. 566 .

16. - J. Haritschelhar, L'ceuvre poétique de Pierre Topet-Etchahun, Bilbao, 1970,

17 - J. Haritschelhar, Le poète souletin Pierre Topet Etchahun (1786-1862), Contribution à l'étude de la poésie populaire basque du XIX' siècle, Amis du Musée basque, Bayonne, 1969, p. 363.

18 - J. Haritschelhar, Le poète souletin Pierre Topet Etchahun (1786-1862), Contribution à l'étude de la poésie populaire basque du XIX'siècle. Amis du Musée basque, Bayonne, 1969, p. 417-436.

19 - J. Haritschelhar, L'ceuvre poétique de Pierre Topet-Etchahun, Bilbao, 1970, p. 378. 